Besonders interessant ist das 6. Kapitel ("Zinsen und riba in einigen arabischen Staaten", S. 133-174), in dem der Verfasser die praktische Behandlung von vertraglichen Zinsvereinbarungen in einigen arabischen Staaten darstellt. Besonders eingehend wird die Lage in den Vereinigten Arabischen Emiraten behandelt (S. 133-149), einen weiteren Schwerpunkt bildet Saudi-Arabien (S. 149-161), daneben finden auch Kuwait (S. 161-166), Bahrain (S. 166-168), Oman (S. 168-171) Qatar (S. 171-172) und Libyen (S. 173-174) Berücksichtigung. Alle Staaten haben auf verschiedenen Wegen versucht, die Bedürfnisse des internationalen Wirtschafts verkehrs, der ohne Zinsvereinbarungen kaum funktionieren würde, mit den islamischrechtlichen Anforderungen zu vereinbaren. Meist werden Handelsverträge direkt oder verdeckt dem Anwendungsbereich des riba-Verbotes entzogen. Vor allem, wenn das verdeckt geschieht, wie etwa in Saudi-Arabien, geht das auf Kosten der Rechtssicherheit. Es erscheint bislang offenbar unmöglich, islamisches Recht und Wirtschaftsleben zu versöhnen.

Abgerundet wird das Werk durch ein arabisch-deutsches Glossar und ein recht ausführliches Literaturverzeichnis. Leider fehlt ein Stichwortverzeichnis, das die Arbeit auch als Nachschlagewerk verwendbar gemacht hätte. Das Werk füllt eine Lücke. Meine kritischen Anmerkungen sollen seine Bedeutung nicht schmälern. Für Praktiker wird vor allem die Darstellung der Rechtslage in einigen arabischen Staaten interessant sein, die durchweg wichtige Handelspartner Deutschlands sind. Jedem, der sich wissenschaftlich mit aktuellen Problemen des riba-Verbotes beschäftigt, bietet es einen hilfreichen Einstieg in die komplexe und nur schwer durchschaubare Materie.

Johannes Christian Wichard

\title{
Hans-Georg Ebert
}

Das Personalstatut arabischer Länder: Problemfelder, Methoden, Perspektiven

Ein Beitrag zum Diskurs über Theorie und Praxis des islamischen Rechts

Leipziger Beiträge zur Orientforschung, Bd. 7

Peter Lang Verlag, Frankfurt am Main, 1996, 187 S., DM 65,--

Ziel des Werkes ist es, "die familienrechtlichen Bestimmungen der arabischen Länder vergleichend" zu analysieren. Einschränkend vermerkt der Autor allerdings, daß "von den 23 Mitgliedern der Arabischen Liga ... Djibouti, die Komoren, Mauretanien, Somalia, Sudan und Palästina sowie die nicht dieser Organisation angehörende Westsahara aus quellenmäßigen, staatsrechtlichen und anderen Gründen nicht oder nur teilweise erfaßt" werden (S. 9). Da die arabischen Staaten im Bereich des Familien- und Erbrechts zumeist nach Konfessionen differenzieren ( $S$. 14 ff.), beschränkt sich die Arbeit auf die für die sunnitischen Muslime geltenden Vorschriften. Auch dann bleibt noch genügend Material, um Bibliotheken zu füllen. Eine Arbeit, die diese Materialfülle sichtet und knapp zusam- 
menstellt, ist in deutscher Sprache sehr willkommen. Umfassendere Darstellungen des Familienrechts liegen bislang vor allem in anderen europäischen Sprachen vor. ${ }^{1}$ Der Titel ist allerdings für deutsche Juristen mißverständlich: Im heutigen deutschen Sprachgebrauch ist der Begriff "Personalstatut" ein Terminus des internationalen Privatrechts und bezeichnet "diejenige Rechtsordnung, welche für alle persönlichen Rechtsverhältnisse eines Menschen oder auch einer juristischen Person oder einer Personengesamtheit maßgebend ist" ${ }^{2}$. Der Verfasser folgt offensichtlich dem französischen Sprachgebrauch, der unter "Personalstatut" nicht nur die zur Regelung der persönlichen Rechtsverhältnisse berufene Rechtsordnung versteht, sondern auch die Gesamtheit der Sachgebiete, die dem Personalstatut unterstehen. ${ }^{3}$ In diesem letzteren Sinne, also als Oberbegriff für alle Normen, die die persönlichen Rechtsverhältnisse ordnen, wird der Begriff auch im arabischen Sprachraum verwendet (S. $11 \mathrm{f}$.).

Das erste Kapitel, "Islamisches Recht im Spannungsverhältnis von Islamisierung und Säkularisierung" (S. 11-39), wendet sich einem zentralen Problem zu. Das Familien- und Erbrecht ist eine der letzten Bastionen des islamischen Rechts; in den meisten arabischen Staaten hat es sich nur hier in Gestalt von "Personalstatutsgesetzen" erhalten. Gleichzeitig ist es in diesen Bereichen stark überformt worden: Schon die Kodifizierung und die damit verbundene Zentralisierung des Rechts in der Hand des Staates bedeutet einen massiven Eingriff. Hinzu kommen eine Vielzahl offener und versteckter Neuerungen, mit denen die Staaten in verschiedenem Maße versucht haben, das Recht zu modernisieren und zu säkularisieren (S. 27 ff.). Diese Relikte des islamischen Rechts sind nunmehr im Zuge der zunehmenden Islamisierung von Politik und Gesellschaft zum Kampfplatz der verschiedenen Richtungen geworden. Einige dieser Konflikte werden am Ende des Kapitels kurz dargestellt.

Im zweiten Kapitel, "Menschenrechte, Grundrechte, Bürgerrechte und Persönlichkeitsrechte in den arabischen Ländern" (S. 41-58), wird ein weiterer neuralgischer Punkt des heutigen islamischen Rechtsverständnisses kurz angesprochen. Der Verfasser beschränkt sich hier auf die für seine Zwecke relevanten Felder der Gleichberechtigung von Mann und Frau und auf die Religionsfreiheit. In beiden Bereichen bestehen nach wie vor Defizite. Immerhin sieht der Verfasser noch Raum für die Emanzipation der Frau (S. 46). Es folgt eine Zusammenstellung der Aussagen zum Personalstatut, die sich in staatlichen Grunddokumenten und internationalen Verträgen der arabischen Staaten finden.

1

Vgl. allerdings Rauscher, Islamisches Familienrecht der sunna und shîa, 1987; vgl. weiter etwa Anderson, Islamic Family Law, International Encyclopedia of Comparative Law, Vol. 4, ch. 4, 1983, S. 55-79; Linant de Bellefonds, Traité de Droit Musulman Comparé, Vol. 2 und 3; Mallat / Connors (eds.), Islamic Family Law, 1990; Nasir, The Islamic Law of Personal Status, 1990.

2

Kropholler, Internationales Privatrecht, 2. Aufl. 1994, S. 236; vgl. auch Deutsches Rechtslexikon, 2. Aufl. 1992, Bd. 2; Köbler, Juristisches Wörterbuch, 7. Aufl. 1995, S. 287.

3

Kropholler, a.a.O. 
Das dritte Kapitel "Das Personalstatut arabischer Länder: Rechtslage und Quellenverweise" (S. 59-88) besteht im wesentlichen aus einer sehr hilfreichen Zusammenstellung der einschlägigen Gesetzestexte mit Hinweisen auf Sekundärliteratur in Arabisch und in europäischen Sprachen. Damit ist die Grundlage für das vierte Kapitel ("Systematischer Teil", S. 89-129) gelegt, das eine vergleichende Darstellung der Gesetzeslage in den Bereichen Eheschließung und Ehewirkungen (S. 89-108), Scheidung (S. 108-118), Verwandtschaft und Vormundschaft (S. 118-129) bringt. Das ist bei weitem der interessanteste Teil des Werkes, da der Verfasser hier neben den einschlägigen Vorschriften immer wieder auch die gesellschaftlichen und sozialen Hintergründe kurz andeutet, wobei er sich zumeist auf neuere Sekundärliteratur stützt. An einigen Stellen sind seine Aussagen allerdings recht pauschal, etwa wenn es allgemein heißt: "Frauen werden auch bei Gericht in Familienangelegenheiten de facto erheblich benachteiligt. Der Richter entscheidet zumeist für den Mann und gegen die Frau" (S. 118), und als Nachweis nur ein englischsprachiger Aufsatz zur Lage der Frauen in Bahrain zitiert wird. Auch wird für den Laien der Unterschied zwischen der klassisch-islamischen und der heutigen Rechtslage nicht immer hinreichend deutlich. So etwa, wenn es heißt: "Jegliche sexuelle Verbindung außerhalb der Ehe ist harâm und unterliegt ggf. der hadd-Strafe für Unzucht (zinâ)" (S. 105). Daß die meisten arabischen Staaten die koranischen Strafen heute nicht mehr anwenden, sondern sich Strafgesetzbücher nach westlichem Vorbild gegeben haben, erfährt man hingegen nicht. Strafbar ist in romanischer Tradition etwa in Algerien oder Tunesien nur noch der Ehebruch, vgl. Art. 339 des tunesischen und Art. 341 des algerischen Strafgesetzbuches. Mißverständlich ist schließlich die Aussage "Vaterschaftsklagen bedürfen im Konfliktfalle der baiyina (Beweis ...)" (S. 120). Sie trifft nur für die gerichtliche Feststellung der ehelichen Vaterschaft zu. Die nichteheliche Vaterschaft hingegen begründet im islamischen Recht keinerlei Rechtswirkungen und kann auch gerichtlich nicht festgestellt werden. Es ist daher zwar vom juristischen Standpunkt aus konsequent, wenn der Verfasser die nichteheliche Kindschaft unberücksichtigt läßt. Ein "Problemfeld" in sozialer und kultureller Hinsicht stellt sie dennoch dar. ${ }^{4}$

Das abschließende fünfte Kapitel ist einigen "Tendenzen der Rechtsentwicklung auf dem Gebiet des Personalstatuts in den arabischen Ländern" gewidmet (S. 131-134). Der Verfasser nennt hier Gründe für die Unterschiede zwischen den einzelnen arabischen Staaten (Rechtsschulen, Gewohnheitsrecht, internationale Verpflichtungen, nichtislamische Traditionen) und unterstreicht noch einmal die Bedeutung religiöser und regionaler Traditionen im Prozeß der "Modernisierung" des Rechtssystems. Überschneidungen, Widersprüche und Gesetzeslücken in den Gesetzestexten werden erklärt als "Mittel, den fehlenden nationalen, religiösen oder politischen Konsens zu verdecken und die Gesetze für unterschiedliche Kräfte akzeptabel zu machen" (S. 132 f.). Wichtig ist auch der Hinweis, daß die Kodifizie-

Vgl. etwa zur Lage in Algerien Aït-Zä̈, L'abandon d'enfant et la loi, RA 29 (1991), 473; Saadi, Le nom, le sang ou la filiation exhortée par le droit, RA 29 (1991), 55; Sarida Zidani, L'enfant ne hors mariage en Algérie, Algier 1991. 
rung "systemkonformes Verhalten aller Bevölkerungsgruppen fördern und 'staatsfreie' Nischen in der Gesellschaft verschließen" soll (S. 133). Mit dem letzten Aspekt schließt sich dann der Kreis wieder: "Das Familienrecht avanciert zum Feld der Auseinandersetzungen islamistischer und zaghaft feministischer Kräfte, zwischen denen der Staat immer weniger auszugleichen vermag" (S. 133). Der Schlußsatz des Werkes läßt dann allerdings vielfältige Deutungen zu: "Die Perspektiven der gültigen Gesetze zum Personalstatut ergeben sich nicht nur aus der Entwicklung der religiösen Verhältnisse, sondern sind eine Funktion verschiedener politischer, sozialer und kultureller Aspekte mit zahlreichen Variablen" (S. 134). Abgerundet wird das Werk durch ein Literaturverzeichnis und ein sehr hilfreiches Glossar islamisch-rechtlicher Termini. Leider fehlt ein Stichwortverzeichnis, das

die Zugänglichkeit des Werkes und seine Verwendbarkeit als Nachschlagewerk deutlich erhöht hätte.

Der Untertitel "Problemfelder, Methoden, Perspektiven" deutet auf eine Eigenheit des Werkes hin: Es enthält eine Vielzahl von Auflistungen und Zusammenstellungen des relevanten Materials, weshalb es nicht ganz leicht lesbar ist. Zudem werden viele Probleme nur kurz angerissen, so daß die Arbeit an manchen Stellen etwas skizzenhaft wirkt. Sie stellt Material zusammen, wertet die Sekundärliteratur aus und wirft einige interessante Fragen auf, ohne sie jedoch ausführlich abzuhandeln. Das ist auf nicht einmal zweihundert Seiten natürlich auch nicht möglich und soll die Verdienste des Werkes auch nicht schmälern: Besonders der "systematische Teil" mit der knappen vergleichenden Darstellung familienrechtlicher Vorschriften arabischer Staaten wird für Wissenschaft und Praxis ein äußerst hilf reicher Einstieg sein, wenn es um Fragen des geltenden Familienrechts der arabischen Staaten geht.

Johannes Christian Wichard

Eve Sandberg (Hrsg.)

The Changing Politics of Non-Governmental Organizations and African States

Praeger, Westport (Connecticut) / London, 1994, 206 S., £ 43,95

Der Sammelband beschäftigt sich in acht Beiträgen mit den Beziehungen zwischen Nichtregierungsorganisationen (NRO) und dem Staat in Afrika. Die Bedeutung der NRO hat seit Ende der 1980er Jahre in Afrika eine zunehmend wichtige Rolle in der Entwicklungszusammenarbeit gespielt. Bis zum Ausbruch der Demokratisierungswelle wurden NRO von den autoritären Regimen als bedrohliche Konkurrenz gesehen und ihre Aktivitäten argwöhnisch verfolgt oder gezielt behindert. Es galt, den Aufbau von vom Staat unabhängigen Organisationen zu verhindern. Damit sollte vermieden werden, daß die Geberländer NRO zunehmend als Kooperationspartner betrachteten und diesen einen Teil der Entwicklungshilfe direkt zur Verfügung stellten. Dies hätte für das autoritäre Regime bedeutet, diese 